\title{
Seleção de sítios de oviposição em anuros (Lissamphibia)
}

\author{
Wagner Rodrigues da Silva ${ }^{1,2}$ \& Ariovaldo Antônio Giaretta ${ }^{1}$ \\ ${ }^{1}$ Laboratório de Taxonomia, Ecologia Comportamental e Sistemática de Anuros Neotropicais, \\ Instituto de Biologia, Campus Umuarama, Universidade Federal de Uberlândia - UFU, \\ Bloco 2d, CEP 38400-902, Uberlândia, MG, Brasil, e-mail: thoropa@inbio.ufu.br \\ ${ }^{2}$ Autor para correspondência: Wagner Rodrigues da Silva, e-mail: wagnerdrigues@yahoo.com.br
}

SILVA, W.R. \& GIARETTA, A.A. Oviposition site selection in anurans (Lissamphibia). Biota Neotrop. 8(2): http://www.biotaneotropica.org.br/v8n3/en/abstract?thematic-review+bn02008032008.

\begin{abstract}
Oviposition site selection is a kind of habitat selection in which the females choose egg-laying sites that maximize their reproductive success. In the present review, we compiled the main conclusions of major studies on this theme that used frogs as model organisms. In the literature, three main lines of investigations were recognized: 1) multiple factor analyses in natural habitats- within the breeding site, places with eggs/tadpoles are compared with unused sites, testing several abiotic and biotic factors that could potentially influence female's choice; 2) experiments with pools arranged in replicated blocks (treatments/control) to simulate oviposition sites differing in quality and 3) quantification of reproductive events associated with experimental manipulations in the field. Effectively, females of several frog species select oviposition sites more favorable to survival and development of eggs and tadpoles. In general, the female choice depends on the abundance and quality of potential egg-laying sites and on her discriminatory ability. Female choices usually mirror the main risks to survival and growth of the offspring, but discriminatory power is not absolute and partial or total losses of clutches and tadpoles may occur, most of them resulting from desiccation and predation. The sensory mechanisms that females use to discriminate the conditions of potential oviposition sites remain poorly known and unexplored. Besides affecting the parental reproductive success, the selective behavior potentially influence population dynamics and community structure by promoting habitat segregation among competitive species or prey-species and their predators.

Keywords: habitat selection, egg-laying microhabitat, reproductive success, indirect effects, methodological approaches.
\end{abstract}

SILVA, W.R. \& GIARETTA, A.A. Seleção de sítios de oviposição em anuros (Lissamphibia). Biota Neotrop.8(2): http://www.biotaneotropica.org.br/v8n3/pt/abstract?thematic-review+bn02008032008.

Resumo: A seleção de sítios de oviposição é uma forma de seleção de hábitat em que as fêmeas escolhem o micro-ambiente de desova procurando maximizar o sucesso reprodutivo. Nesta revisão, compilamos as principais conclusões de estudos sobre seleção de sítios de oviposição tendo os anuros como modelo. Na literatura, podemos reconhecer três linhas principais de investigação: 1) análise de múltiplos fatores em hábitat natural- no ambiente de reprodução, sítios com ovos e girinos são comparados com locais não utilizados com base em fatores abióticos e bióticos com potencial de influenciar a escolha da fêmea; 2) experimentos controlados com poças dispostas em blocos replicados simulando sítios potenciais de oviposição com diferenças na qualidade e 3) quantificações de eventos reprodutivos e experimentos de manipulação em ambiente natural. Fêmeas de várias espécies de anuros selecionam sítios de oviposição mais favoráveis à sobrevivência e desenvolvimento dos ovos e girinos. O comportamento de escolha depende da abundância e qualidade relativa dos locais potenciais de desova e da capacidade de discriminação da fêmea. Em geral, as escolhas das fêmeas respondem aos principais riscos à sobrevivência e desempenho da prole, porém apresentam eficiência relativa e perdas parciais ou totais de desovas e girinos são ocasionadas, principalmente, por fatores de mortalidade direta como dessecação e predação. Os mecanismos sensoriais que elas utilizam para discriminar as condições do ambiente de reprodução ainda são praticamente desconhecidos. Além de afetar o sucesso reprodutivo dos parentais, o comportamento de escolha pode influenciar na dinâmica de populações e estrutura de comunidades ao promover a segregação de hábitat entre espécies competidoras ou entre espécies-presa e seus predadores.

Palavras-chave: seleção de hábitat, micro-ambiente de desova, sucesso reprodutivo, efeitos indiretos, métodos de estudo. 


\section{Introdução}

A seleção de hábitat é um processo pelo qual indivíduos escolhem ambientes mais favoráveis à sobrevivência e reprodução (revisão em Morris 2003). A seleção de sítios de oviposição é uma forma de seleção de hábitat em que os parentais, particularmente as fêmeas, escolhem o micro-ambiente de desova. Esse comportamento afeta o sucesso reprodutivo individual porque estabelece as condições de sobrevivência e desenvolvimento dos ovos e larvas. Portanto, a seleção natural deve favorecer fêmeas capazes de escolher locais de desova que maximizem o sucesso reprodutivo por meio de uma maior taxa de sobrevivência da prole (Bernardo 1996, Resetarits-Jr. 1996, Mousseau \& Fox 1998).

Os anuros têm sido amplamente utilizados como modelo em estudos sobre seleção de sítios de oviposição (e.g., Skelly 2001, Binckley \& Resetarits-Jr. 2002, 2003, Murphy 2003a, b, Rieger et al. 2004, Rudolf \& Rödel 2005, Goldberg et al. 2006, Halloy 2006, Haramura 2008, Lin \& Kam 2008). Eles constituem bons modelos nesse tema porque apresentam modos reprodutivos variados, reproduzem-se em hábitats diversos, são relativamente conspícuos devido à vocalização, concentram-se em pequenas áreas no período reprodutivo, desovam em locais espacialmente definidos (e.g., poças) e a maioria possui uma fase larval que se desenvolve nas condições definidas pelos adultos (Duellman \& Trueb 1994, Murphy 2003a, Marsh \& Borrell 2001, Rudolf \& Rödel 2005).

Nesta revisão, compilamos as principais conclusões de estudos sobre seleção de sítios de oviposição em anuros (Anura) visando estabelecer comparações e destacar possíveis padrões e mecanismos gerais desse comportamento. Os dados obtidos através desse táxon podem contribuir para o esclarecimento de aspectos ecológicos e evolutivos da seleção de sítios de oviposição (e.g., principais pressões seletivas, efeitos indiretos nas populações e comunidades) e também para a orientação de linhas gerais de futuras pesquisas.

\section{Métodos de Estudo}

$\mathrm{Na}$ literatura, podemos reconhecer três linhas principais de investigação sobre seleção de sítios de oviposição:

1) Análise de múltiplos fatores em hábitat natural: no ambiente de reprodução da espécie em questão, sítios com ovos e girinos são comparados com sítios não utilizados. Fatores abióticos e bióticos com potencial de influenciar a escolha da fêmea são analisados simultaneamente (e.g., profundidade, propriedades físico-químicas da água, presença e tipo de competidores, predadores). Para inferir se há escolha de locais de postura específicos, os estudos analisam se a distribuição das desovas no ambiente tem associação com alguns desses fatores e se existe preferência ou se determinadas condições são evitadas (e.g., Howard 1978, Seale 1982, Caldwell 1986, Banks \& Beebee 1987, Holomuzki 1995, Evans et al. 1996, Kam et al. 1996, Laurila 1998, Mitchell 2002, Dougherty et al. 2005, Ficetola et al. 2006, Goldberg et al. 2006, Hagman \& Shine 2006, Lin \& Kam 2008).

2) A segunda abordagem consiste em experimentos controlados em cativeiro ou semi-cativeiro (poças artificiais introduzidas em ambiente natural). Poças artificiais (e.g., recipientes plásticos) são dispostas de forma replicada (aos pares ou em blocos) para simular sítios potenciais de oviposição com diferenças nos fatores investigados (e.g., predador presente vs. ausente). Os tratamentos normalmente correspondem a sítios de baixa qualidade por oferecer, por exemplo, riscos de dessecação, competição, parasitismo, predação ou diferentes combinações e intensidades desses fatores. Os controles não apresentam esses fatores adversos e seriam, portanto, mais favoráveis à sobrevivência e desenvolvimento dos ovos e girinos. O comportamento das fêmeas é avaliado com base na distribuição dos ovos e girinos entre os tratamentos (ou controle). O objetivo principal do estudo é distinguir se a distribuição dos ovos é aleatória ou se as fêmeas são capazes de selecionar sítios de melhor qualidade (e.g., Resetarits-Jr. \& Wilbur 1989, Crump 1991, Hopey \& Petranka 1994, Petranka et al. 1994, Laurila \& Aho 1997, Dillon \& Fiaño 2000, Binckley \& Resetarits Jr. 2002, 2003, Rieger et al. 2004, Halloy 2006, Haramura 2008); e

3) Alguns trabalhos têm procurado associar quantificações de eventos reprodutivos e experimentos de manipulação no ambiente natural. Nesta abordagem, o pesquisador determina alguns fatores-chave que afetam o sucesso reprodutivo da espécie em questão e depois avalia se esses fatores de fato influenciam a distribuição espacial e temporal das desovas. Para que o estudo não se limite a análises de correlação, experimentos podem ser realizados para atestar se há relação causal entre os fatores analisados e as escolhas das fêmeas. Aqui o objetivo principal é determinar se a escolha da fêmea quanto ao momento e local de postura reflete os principais riscos à sobrevivência e desenvolvimento dos ovos e girinos (e.g., Spieler \& Linsenmair 1997, Marsh \& Borrell 2001, Murphy 2003a, b, Rudolf \& Rödel 2005).

\section{Compilação Bibliográfica}

Em geral, os estudos têm demonstrado que fêmeas de diversas espécies de anuros de diferentes grupos selecionam sítios de oviposição mais favoráveis à sobrevivência e desenvolvimento de seus ovos e girinos (e.g., Skelly 2001, Binckley \& Resetarits-Jr. 2002, 2003, Murphy 2003a, b, Rieger et al. 2004, Rudolf \& Rödel 2005, Goldberg et al. 2006, Halloy 2006, Haramura 2008). Dentre aproximadamente 35 estudos de caso publicados envolvendo 24 espécies, apenas em Rana temporaria Linnaeus, 1758 (Ranidae), a fêmea parece não ser seletiva em relação à presença de predadores de seus girinos (e.g., larvas de insetos, peixes) (Laurila \& Aho 1997, Laurila 1998). Dentre esses estudos, compilamos na Tabela 1 os dados de publicações com quantificações mais robustas, experimentos elaborados e conclusões generalizadas sobre o assunto. Nela, apresentamos sucintamente as respostas das fêmeas, segundo a interpretação dos autores, frente aos principais fatores adversos à sobrevivência e desenvolvimento dos ovos e girinos. Destacamos que, no presente momento, em decorrência do número ainda insuficiente de publicações, da diversidade de linhagens (e.g., famílias), de fatores (e.g., tipo de predador), contextos (e.g., biomas, hábitats) e métodos dos diferentes estudos, ainda não é possível uma avaliação formal quantitativa (estatística) de eventuais padrões e tendências gerais.

A seleção de sítios de oviposição nos anuros parece envolver reconhecimento e discriminação de características estruturais e físicoquímicas do corpo d'água (e.g., tamanho, profundidade, temperatura, pH) e/ou evitação de fatores de risco como dessecação, competição, parasitismo, canibalismo e predação (referências: Tabela 1). Os autores interpretaram a seleção como comportamentos deliberados de preferência ou evitação de determinadas condições no sentido de favorecer o sucesso reprodutivo individual. A escolha depende da abundância e qualidade relativa dos sítios, da capacidade de discriminação das fêmeas e da competição entre elas por locais de postura (Binckley \& Resetarits Jr. 2003, Murphy 2003a, Rudolf \& Rödel 2005). No geral, as fêmeas parecem reconhecer e discriminar diferenças sutis entre micro-ambientes similares (e.g., Kam et al. 1996, Rudolf \& Rödel 2005, Ficetola et al. 2006, Haramura 2008, Lin \& Kam 2008). No entanto, os mecanismos sensoriais que elas 
Table 1. The major studies about oviposition site selection in anurans (Anura). The focal species, field/experimental conditions, factors that affect survival of eggs/tadpoles and authors' interpretation on female responses to these factors are presented for each publication. Updated and modified from Skelly (2001).

Tabela 1. Os principais estudos sobre seleção de sítios de oviposição em anuros (Anura). A espécie estudada, condições de campo e experimentais, fatores significativos que afetam a sobrevivência de ovos/girinos e interpretação dos autores em relação às respostas das fêmeas frente a esses fatores estão apresentados para cada publicação. Atualizado e modificado de Skelly (2001).

\begin{tabular}{|c|c|c|c|c|}
\hline Fonte & Espécie & Condições & Fatores analisados & Interpretação \\
\hline Resetarits Jr. \& Wilbur (1989) & $\begin{array}{l}\text { Hyla } \\
\text { chrysoscelis }\end{array}$ & $\begin{array}{l}\text { Poças artificiais } \\
\text { (plásticas) }\end{array}$ & $\begin{array}{l}\text { Girinos co-específicos, teleósteos, } \\
\text { larvas de salamandra, girinos } \\
\text { heteroespecíficos, salamandras } \\
\text { adultas e insetos }\end{array}$ & $\begin{array}{l}\text { Evitar competição } \\
\text { Evitar predação } \\
\text { Nenhum efeito }\end{array}$ \\
\hline Crump (1991) & $\begin{array}{l}\text { Isthmohyla } \\
\text { pseudopuma }\end{array}$ & Poças artificiais & $\begin{array}{l}\text { Girinos coespecíficos canibais, } \\
\text { profundidade da poça }\end{array}$ & $\begin{array}{l}\text { Evitar predação } \\
\text { Evitar dessecação }\end{array}$ \\
\hline Hopey \& Petranka (1994) & $\begin{array}{l}\text { Lithobates } \\
\text { sylvaticus }\end{array}$ & $\begin{array}{l}\text { Poças escavadas } \\
\text { (campo) }\end{array}$ & Teleósteos predadores de ovos & Evitar predação \\
\hline Petranka et al. (1994) & $\begin{array}{l}\text { Anaxyrus } \\
\text { americanus }\end{array}$ & Poças escavadas & $\begin{array}{l}\text { Girinos heteroespecíficos } \\
\text { predadores de ovos e girinos }\end{array}$ & Evitar predação \\
\hline Laurila \& Aho (1997) & $\begin{array}{l}\text { Rana } \\
\text { temporaria }\end{array}$ & $\begin{array}{l}\text { Poças em } \\
\text { hábitat natural }\end{array}$ & Teleósteos predadores de girinos & Nenhum efeito \\
\hline Spieler \& Linsenmair (1997) & $\begin{array}{l}\text { Hoplobatrachus } \\
\text { occipitalis }\end{array}$ & $\begin{array}{l}\text { Poças em } \\
\text { hábitat natural }\end{array}$ & $\begin{array}{l}\text { Girinos co-específicos canibais, } \\
\text { teleósteos, estabilidade hídrica } \\
\text { da poça }\end{array}$ & $\begin{array}{l}\text { Evitar predação } \\
\text { Evitar dessecação }\end{array}$ \\
\hline Fegraus \& Marsh (2000) & $\begin{array}{l}\text { Engystomops } \\
\text { pustulosus }\end{array}$ & Poças artificiais & $\begin{array}{l}\text { Idade da poça e propriedades } \\
\text { físico-químicas da água }\end{array}$ & Nenhum efeito \\
\hline Kiesecker \& Skelly (2000) & $\begin{array}{l}\text { Hyla } \\
\text { versicolor }\end{array}$ & Poças artificiais & $\begin{array}{l}\text { Caramujos infectados } \\
\text { com trematódeos parasitas }\end{array}$ & Evitar parasitismo girinos \\
\hline Marsh \& Borrel (2001) & $\begin{array}{l}\text { Engystomops } \\
\text { pustulosus }\end{array}$ & $\begin{array}{l}\text { Poças artificiais } \\
\text { Riacho natural }\end{array}$ & $\begin{array}{l}\text { Presença de ninhos e } \\
\text { girinos co-específicos na poça }\end{array}$ & $\begin{array}{l}\text { Evitar coespecíficos } \\
\text { Preferência pela } \\
\text { presença de coespecíficos }\end{array}$ \\
\hline Binckley \& Resetarits Jr. (2002) & $\begin{array}{l}\text { Hyla } \\
\text { squirella }\end{array}$ & Poças artificiais & Teleósteos predadores de ovos & Evitar predação \\
\hline Binckley \& Resetarits Jr. (2003) & $\begin{array}{l}\text { Hyla } \\
\text { chrysoscelis }\end{array}$ & Poças artificiais & Teleósteos predadores de ovos & Evitar predação \\
\hline Murphy (2003a) & $\begin{array}{l}\text { Edalorhina } \\
\text { perezi }\end{array}$ & $\begin{array}{l}\text { Poças em } \\
\text { hábitat natural }\end{array}$ & $\begin{array}{l}\text { Estabilidade hídrica da poça, } \\
\text { náiades de Odonata } \\
\text { predadoras de girinos, } \\
\text { presença de ninhos e } \\
\text { girinos co-específicos na poça }\end{array}$ & $\begin{array}{l}\text { Evitar dessecação } \\
\text { Evitar predação } \\
\text { Evitar competição }\end{array}$ \\
\hline Murphy (2003b) & $\begin{array}{l}\text { Edalorhina } \\
\text { perezi }\end{array}$ & Poças artificiais & $\begin{array}{l}\text { Náiades de Odonata e presença } \\
\text { de girinos co-específicos }\end{array}$ & $\begin{array}{l}\text { Evitar predação } \\
\text { Evitar competição }\end{array}$ \\
\hline Rieger et al. (2004) & $\begin{array}{l}\text { Hyla } \\
\text { femoralis }\end{array}$ & Poças artificiais & Teleósteos predadores de girinos & Evitar predação \\
\hline Rudolf \& Rödel (2005) & $\begin{array}{l}\text { Phrynobatrachus } \\
\text { guineensis }\end{array}$ & $\begin{array}{l}\text { Hábitat natural } \\
\text { Experimentos } \\
\text { (campo) }\end{array}$ & $\begin{array}{l}\text { Estabilidade hídrica da } \\
\text { poça e presença de girinos } \\
\text { co-específicos }\end{array}$ & $\begin{array}{l}\text { Evitar dessecação } \\
\text { Preferência pela } \\
\text { presença de coespecíficos }\end{array}$ \\
\hline
\end{tabular}

utilizam para discriminar as condições de sítios potenciais de oviposição ainda são praticamente desconhecidos. Spieler \& Linsenmair (1997) sugeriram que fêmeas de Hoplobatrachus occipitalis (Gunther, 1858) (Dicroglossidae) avaliam a presença, densidade e tamanho de girinos coespecíficos através do olfato.

\section{Estudos de Caso}

Fêmeas de Lithobates catesbeianus (Shaw, 1802) (Ranidae) preferem desovar em sítios defendidos por machos maiores, onde seus embriões se desenvolvem mais rapidamente (água mais quente) e são menos atacados por sanguessugas. Portanto, a territorialidade parece estar relacionada ao domínio de locais de postura de maior qualidade e a predação por sanguessugas deve ser a principal pressão no processo de escolha (Howard 1978).

Lithobates sylvaticus (LeConte, 1825) desova comunalmente e prefere poças maiores e micro-ambientes com água mais quente, provavelmente porque essas condições aceleram o desenvolvimento dos girinos e reduzem os riscos de dessecação (Seale 1982). Lithobates sphenocephalus (Cope, 1886) apresenta desovas comunais apenas na estação fria (Caldwell 1986). Portanto, desova comunal deve conferir um micro-ambiente termicamente favorável aos ovos (e.g., acelerar o desenvolvimento) de espécies de Lithobates que se reproduzem em poças temporárias em regiões frias (Caldwell 1986).

Em condições experimentais, Zina (2006) observou que fêmeas de Engystomops pustulosus (Cope, 1864) (Leiuperidae) preferem 
desovar comunalmente, o que parece reduzir os riscos de dessecação de ovos e girinos. Desovas comunais ocorrem em outras espécies de Leiuperidae (e.g., Eupemphix nattereri Steindachner, 1863, Physalaemus marmoratus (Reinhardt e Lutken, 1862)), porém, ainda não foi demonstrado se este fenômeno é gerado pelo comportamento de escolha efetiva das fêmeas. Outra distinção importante seria determinar se desovas comunais ocorrem porque as fêmeas preferem desovar em sítios já ocupados por desovas coespecíficas ou representam apenas um efeito colateral da escolha de sítios de maior qualidade por várias fêmeas com base em outros fatores ambientais (Giaretta \& Menin 2004, Giaretta \& Facure 2006). Portanto, estudos adicionais serão necessários para comprovar se este fenômeno é gerado pelo comportamento de escolha efetiva das fêmeas e quais as vantagens e desvantagens associadas.

Lithobates sylvaticus e L. palustris (LeConte, 1825) preferem hábitats sem peixes (Hopey \& Petranka 1994, Holomuzki 1995), porém Rana temporaria (Ranidae) parece não evitar a presença de larvas de insetos ou peixes predadores de seus girinos (Laurila \& Aho 1997, Laurila 1998). Em R. japonica Boulenger, 1879, a escolha de locais de postura parece envolver um balanço entre tamanho do corpo d'água e competição intra-específica. Entre poças pequenas, as fêmeas evitam aquelas com ovos e girinos coespecíficos, o que não ocorre entre poças maiores (Matsushima \& Kawata 2005).

Bufo bufo (Linnaeus, 1758) e Epidalea calamita (Laurenti, 1768) (Bufonidae) preferem poças maiores apesar de conterem peixes predadores, provavelmente porque seus girinos são impalatáveis (Banks \& Beebee 1987, Laurila 1998). No entanto, Anaxyrus americanus (Holbrook, 1836) (Bufonidae) escolhe poças mais efêmeras, porém sem peixes (Holomuzki 1995), e evitam também girinos de Lithobates sylvaticus, os quais são predadores de seus ovos e girinos (Petranka et al. 1994). Em Rhinella marina (Linnaeus, 1758) (Bufonidae), características estruturais e físico-químicas do corpo d'água parecem ser mais importantes que a presença de insetos e peixes predadores no processo de escolha. Poças temporárias, rasas, menos ácidas, com água transparente e sem vegetação marginal são preferidas, provavelmente porque essas condições aceleram o desenvolvimento dos girinos (Evan et al. 1996, Hagman \& Shine 2006).

Os hilídeos (Hylidae) serviram de modelo em estudos experimentais. Em geral, os testes demonstraram que as fêmeas são realmente capazes de selecionar sítios de oviposição mais favoráveis à sobrevivência e desenvolvimento de seus ovos e girinos. Nos experimentos, Hyla chrysoscelis Cope, 1880, H. squirella Bosc in Daudin, 1800 e H.femoralis Bosc in Daudin, 1800 (Hylidae) evitaram poças com peixes, distribuindo a maioria (até 95\%) de suas desovas em situação-controle (sem peixes). Como tendência geral, as fêmeas parecem não discriminar entre níveis contínuos de densidade desses predadores, mas apenas as condições de presença ou ausência, as quais produzem diferenças significativas na sobrevivência dos girinos (Resetarits-Jr. \& Wilbur 1989, Binckley \& Resetarits-Jr. 2002, 2003, Rieger et al. 2004).

As fêmeas de espécies de Hylidae respondem a outros fatores adversos além da predação por peixes. Por exemplo, Isthmohyla pseudopuma (Gunther, 1901) reduz os riscos de canibalismo e dessecação ao preferir poças mais profundas e sem girinos coespecíficos. A escolha resulta de um balanço, porque, no início da noite, poças profundas são favorecidas, porém quando elas se saturam de ovos, a preferência muda para poças rasas com menos ovos (Crump 1991). Fêmeas de $H$. versicolor LeConte, 1825 reconhecem a presença de caramujos portadores de trematódeos parasitas e escolhem poças sem esses moluscos ou com poucos indivíduos não infectados, reduzindo, assim, os riscos de parasitismo de seus girinos (Kiesecker \& Skelly 2000).
Os autores desses estudos experimentais com os hilídeos ressaltam que a seleção de sítios de oviposição pode influenciar na dinâmica de populações e na estrutura de comunidades, promovendo, por exemplo, a segregação de hábitat entre espécies competidoras ou entre espécies-presa e seus predadores. Assim, a ausência de uma espécie num determinado local pode ser atribuída não somente a efeitos diretos (mortalidade) da dessecação, competição, parasitismo ou predação, mas também, ao comportamento de evitação da fêmea a esses fatores adversos (Resetarits-Jr. \& Wilbur 1989, Crump 1991, Hopey \& Petranka 1994, Kiesecker \& Skelly 2000, Binckley \& Resetarits-Jr. 2002, 2003, Rieger et al. 2004, Resetarits Jr. 2005).

Alguns pesquisadores analisaram a seleção de sítios de oviposição em ambiente natural associando experimentos de manipulação das condições locais (e.g., Spieler \& Linsenmair 1997, Marsh \& Borrel 2001, Murphy 2003a, b, Rudolf \& Rödel 2005). Espécies que põem seus ovos em ninhos de espuma flutuantes (muitas espécies de Leiuperidae e Leptodactylidae) foram utilizadas como modelo em alguns desses estudos (e.g., Halloy \& Fiaño 2000, Marsh \& Borrell 2001, Murphy 2003a, b). Os autores investigaram se as escolhas dos parentais refletiam os principais riscos à sobrevivência dos ovos e girinos. Os experimentos testavam se havia evidência de relação causal entre os fatores analisados e as respostas das fêmeas. Em geral, esses estudos (detalhados logo abaixo) demonstraram que a seleção envolve um balanço entre fatores físicos e bióticos e que os parentais podem adotar diferentes estratégias em resposta a variações ambientais nas escalas espacial e temporal (e.g., variação sazonal na densidade de predadores, riscos de dessecação), embora as escolhas nem sempre assegurem proteção absoluta aos ovos e girinos (Spieler \& Linsenmair 1997, Marsh \& Borrell 2001, Murphy 2003a, b). Fatores de mortalidade de ovos e larvas, tais como dessecação e predação, causam maior impacto no sucesso reprodutivo dos parentais comparado à competição ou características estruturais e físico-químicas do corpo d'água. Portanto, é provável que fatores de mortalidade direta sejam as principais pressões sobre a evolução de mecanismos sensoriais que permitam acessar a qualidade do ambiente de reprodução (Spieler \& Linsenmair 1997, Murphy 2003a, b, Rudolf \& Rödel 2005).

A idade da poça e propriedades químicas da água não influenciam na escolha de sítios por Engystomops pustulosus (Leiuperidae) (Fegraus \& Marsh 2000). Em experimentos, os parentais evitam poças com ninhos e girinos coespecíficos, porém, em riachos naturais, poças ocupadas por coespecíficos são selecionadas, provavelmente porque estes indicam condições favoráveis (Dillon \& Fiaño 2000, Marsh et al. 2000, Marsh \& Borrell 2001). Como o aumento da densidade retarda o crescimento dos girinos, a evitação de coespecíficos pode ser uma estratégia vantajosa em experimentos cujo contexto homogêneo não oferece outros fatores adversos a serem considerados pela fêmea. No riacho, a aparente preferência por sítios ocupados provavelmente representa uma resposta à qualidade do hábitat e não à presença de coespecíficos, visto que poças maiores e com mais folhas são as mais utilizadas. Portanto, a seleção de sítios de oviposição nesta espécie envolve um balanço entre fatores físicos e bióticos e os parentais podem adotar estratégias opostas em função do contexto ecológico (Marsh \& Borrell 2001).

A dessecação e predação por larvas de insetos são as principais ameaças ao sucesso reprodutivo de Edalorhina perezi Jimenez de la Espada, 1870 (Leiuperidae) em seu hábitat natural (Murphy 2003a). Os adultos respondem parcialmente a esses fatores na distribuição de suas desovas no ambiente. Por exemplo, poças recém-formadas logo após a incidência de chuvas possuem menos insetos predadores (e.g., náiades de Odonata) e são ativamente selecionadas como sítios de vocalização e oviposição. Até meados da estação chuvosa, as fêmeas 
escolhem poças com maior persistência hídrica, porém, no final, poças efêmeras são as mais utilizadas apesar de oferecerem maiores riscos de dessecação. A presença de coespecíficos retarda o crescimento dos girinos, aumentando os riscos de morte por dessecação, porém, a competição intra-específica parece ser secundária na seleção em relação à predação (Murphy 2003a).

Em experimentos conduzidos no campo, os adultos de E. perezi também escolhem poças sem insetos predadores como sítios de vocalização e oviposição, mas as fêmeas são mais seletivas e evitam também girinos coespecíficos (Murphy 2003b). Ambos os sexos preferem tratamentos com os riscos de competição intra-específica em vez de predação, resposta condizente com o maior impacto da predação na sobrevivência dos ovos e girinos em condições naturais (Murphy 2003a, b). As escolhas das fêmeas exibem variação sazonal, porque, no início da estação chuvosa, elas evitam poças com predadores, mas no final não, provavelmente porque a densidade deles diminui no decorrer da estação ou porque elas tornam-se menos seletivas em razão da aproximação do final do período reprodutivo. No entanto, o esclarecimento deste fenômeno requer mais estudos. As fêmeas podem atrasar a postura quando todos os tratamentos oferecem riscos (competição/ predação) (Murphy 2003b). Esse atraso pode ser vantajoso, porque eventos de secagem completa do corpo d'água eliminam coespecíficos e predadores aquáticos, tornando as poças recém-formadas mais favoráveis à oviposição (Murphy 2003a). Portanto, as escolhas das fêmeas de $E$. perezi respondem parcialmente aos principais riscos à sobrevivência de sua prole e se ajustam a variações ambientais nas escalas espacial e temporal, mas essa capacidade de resposta é limitada e, frequentemente, resulta em perdas parciais ou totais de desovas e girinos (Murphy 2003a, b).

Dessecação e canibalismo são os principais riscos à sobrevivência de girinos de Hoplobatrachus occipitalis (Dicroglossidae). A seleção de sítios de oviposição nesta espécie reflete esses riscos, porque as fêmeas escolhem poças mais persistentes e sem ovos ou girinos coespecíficos. Em experimentos no ambiente natural, elas são capazes de reconhecer também diferenças na densidade e classes de tamanho de girinos co-específicos, porque poças com menores densidades ou aquelas com girinos menores são escolhidas. Provavelmente, elas analisam a estabilidade das poças monitorando a redução no volume de água em visitas consecutivas. Como os experimentos excluíam a possibilidade de avaliações táteis e visuais (tela protetora), é provável então que as fêmeas reconhecessem a presença, densidade e tamanho dos girinos usando o olfato (Spieler \& Linsenmair 1997).

Phrynobatrachus guineensis Guibe e Lamotte, 1962 (Phrynobatrachidae) desova em fitotelmatas ou conchas vazias de moluscos. Os adultos selecionam sítios para vocalização e oviposição com maior capacidade de retenção de água, reduzindo, assim, os riscos de dessecação dos girinos. Provavelmente, eles monitoram a redução no volume de água em visitas consecutivas antes da escolha definitiva. No entanto, muitos sítios com estabilidade hídrica favorável à oviposição não são utilizados, porque as fêmeas preferem aqueles já ocupados por ovos e girinos coespecíficos, os quais devem indicar ausência ou baixa densidade de predadores. Portanto, a seleção de sítios de oviposição em $P$. guineensis envolve um balanço entre fatores abióticos e bióticos. A estabilidade hídrica define inicialmente sítios propícios à oviposição, enquanto que a presença de coespecíficos explica as diferenças na freqüência de uso dentre esses sítios inicialmente favoráveis (Rudolf \& Rödel 2005).

\section{Conclusões Gerais}

Neste tópico, destacamos alguns padrões gerais da seleção de sítios de oviposição e apresentamos ao final algumas questões com potencial para serem exploradas em estudos futuros. A seleção efetiva de sítios de oviposição ocorre em espécies de anuros de diferentes grupos e em hábitats variados e, provavelmente, evoluiu em resposta a pressões seletivas diversas (ver Tabela 1). Em geral, os parentais possuem a capacidade de reconhecer, discriminar e selecionar microsítios mais favoráveis à sobrevivência e desenvolvimento dos ovos e girinos, procurando maximizar, portanto, o sucesso reprodutivo individual. A seleção é realizada essencialmente pelas fêmeas, mas, em algumas espécies (e.g., Edalorhina perezi, Lithobates catesbeianus, Phrynobatrachus guineensis), os machos também participam do processo ao escolherem sítios de vocalização com condições favoráveis à oviposição (e.g., predadores ausentes), porém, mesmo nestes casos, eles são menos seletivos (e.g., indiferentes à presença de girinos coespecíficos).

No ambiente natural, a seleção de sítios de oviposição ocorre em contexto ecológico heterogêneo, variável no tempo e no espaço e envolve um balanço entre fatores abióticos e bióticos. Os fatores que causam mortalidade direta dos ovos e girinos, tais como dessecação e predação, parecem ter maior impacto no processo de escolha em comparação a outros fatores como, competição ou características estruturais e físico-químicas do corpo d'água.

De maneira geral, as escolhas das fêmeas refletem os principais riscos à sobrevivência e desenvolvimento dos ovos e girinos, normalmente apresentam flexibilidade em função do contexto ecológico e promovem um aumento potencial do sucesso reprodutivo. No entanto, as escolhas apresentam eficiência relativa e não são raros os eventos de perda parcial ou total de desovas e girinos.

Além de afetar o sucesso reprodutivo dos parentais, a seleção de sítios de oviposição tem potencial para influenciar a dinâmica de populações e a estrutura de comunidades ao promover a segregação ou co-ocorrência de determinadas espécies. Portanto, é importante considerar esse comportamento em estudos de ecologia de populações e comunidades.

Desovas comunais ocorrem em algumas espécies das famílias Ranidae (e.g., Lithobates sylvaticus, L. sphenocephalus) e Leiuperidae (e.g., Eupemphix nattereri), porém, ainda não foi demonstrado se este fenômeno é gerado pelo comportamento de escolha efetiva das fêmeas.

Um aspecto ainda pouco explorado refere-se aos mecanismos sensoriais que as fêmeas utilizam para reconhecer e discriminar as condições do ambiente de reprodução. Novamente, os anuros podem servir de modelo para esclarecer a evolução desses mecanismos que possibilitam aos organismos selecionarem sítios de oviposição mais favoráveis ao seu sucesso reprodutivo.

Os anuros têm sido amplamente utilizados como modelo em estudos sobre seleção de sítios de oviposição. No entanto, espécies com desova terrestre e girinos aquáticos ainda não foram devidamente exploradas, o que seria pertinente uma vez que as fases iniciais do desenvolvimento ocorrem em ambientes totalmente diferentes daqueles onde os girinos se desenvolvem. Considerando as diferenças entre os ambientes terrestre e aquático nos elementos abiótico e biótico, é provável então que as condições favoráveis para desovas terrestres e girinos aquáticos sejam de naturezas diversas, o que deve exigir das fêmeas critérios diferenciados no processo de escolha (e.g., micro-ambiente sombreado para desovas- proteção contra dessecação versus corpo d'água iluminado para os girinos- acelerar o desenvolvimento larval).

\section{Agradecimentos}

Suporte financeiro pela CAPES (WRS), FAPEMIG e CNPq (AAG). 


\section{Referências Bibliográficas}

BANKS, B. \& BEEBEE, T.J.C. 1987. Factors influencing breeding site choice by the pioneering amphibian Bufo calamita. Holarctic. Ecol. 10(1):14-21.

BERNARDO, J. 1996. Maternal effects in animal ecology. Amer. Zool. 36(2):83-105

BINCKLEY, C.A. \& RESETARITS Jr., W.J. 2002. Reproductive decisions under threat of predation: squirrel treefrog (Hyla squirella) responses to banded sunfish (Enneacanthus obesus). Oecologia 130(1):157-161.

BINCKLEY, C.A. \& RESETARITS Jr., W.J. 2003. Functional equivalence of non-lethal effects: generalized fish avoidance determines distribution of gray treefrog, Hyla chrysoscelis, larvae. Oikos 102(3):623-629.

CALDWELL, J.P. 1986. Selection of egg deposition sites: a seasonal shift in the southern leopard frog, Rana sphenocephala. Copeia 1986(1):249-253.

CRUMP, M.L. 1991. Choice of oviposition site and egg load assessment by a treefrog. Herpetologica 47(3):308-315.

DILLON, M.E. \& FIAÑO, J. 2000. Oviposition site selection by the túngara frog (Physalaemus pustulosus). Copeia 2000(3):883-885.

DOUGHERTY, C.K., VAALA, D.A. \& SMITH, G.R. 2005. Within-pond oviposition site selection in two spring-breeding amphibians (Ambystoma maculatum and Rana sylvatica). J. Freshwater. Ecol. 20(4):781-782.

DUELLMAN, W.E. \& TRUEB, L. 1994. Biology of amphibians. Johns Hopkins University, Baltimore

EVANS, M., YÁBER, C. \& HERO, J.M. 1996. Factors influencing choice of breeding site by Bufo marinus in its natural habitat. Copeia 1996(4):904-912.

FEGRAUS, E.H. \& MARSH, D.M. 2000. Are newer ponds better? Pond chemistry, oviposition site selection, and tadpole performance in the túngara frog, Physalaemus pustulosus. J. Herpetol. 34(3):455-459.

FICETOLA, G.F., VALOTA, M. \& BERNARDI, F. 2006. Temporal variability of spawning site selection in the frog Rana dalmatina: consequences for habitat management. Anim. Biodivers. Conserv. 29(2):157-163.

GIARETTA, A.A. \& MENIN, M. 2004. Reproduction, phenology and mortality sources of a species of Physalaemus (Anura: Leptodactylidae). J. Nat. Hist. 38(13):1711-1722

GIARETTA, A.A. \& FACURE, K.G. 2006. Terrestrial and communal nesting in Eupemphix nattereri (Anura, Leiuperidae): interactions with predators and pond structure. J. Nat. Hist. 40(44-46):2577-2587.

GOLDBERG, F.J., QUINZIO, S. \& VAIRA, M. 2006. Oviposition site selection by the toad Melanophryniscus rubriventris in an unpredictable environment in Argentina. Can. J. Zool. 84(5):699-705.

HAGMAN, M. \& SHINE, R. 2006. Spawning site selection by feral cane toads (Bufo marinus) at an invasion front in tropical Australia. Austral. Ecol. 31(5):551-558.

HALLOY, M. \& FIAÑO, J.M. 2000. Oviposition site selection in Pleurodema borellii (Anura, Leptodactylidae) may be influenced by tadpole presence. Copeia 2000(2):606-609.

HALlOY, M. 2006. Choice of oviposition site in Pleurodema borellii (Leptodactylidae): importance of conspecific tadpole size. S. Am. J. Herpetol. 1(1):72-78.

HARAMURA, T. 2008. Experimental test of spawning site selection by Buergeria japonica (Anura: Rhacophoridae) in response to salinity level. Copeia 2008(1):64-67.

HOLOMUZKI, J.R. 1995. Oviposition sites and fish-deterrent mechanisms to two stream anurans. Copeia 1995(3):607-613.

HOPEY, M.E. \& PETRANKA, J.W. 1994. Restriction of wood frogs to fish-free habitats: how important is adult choice? Copeia 1994(4):1023-1025.

HOWARD, R.D. 1978. The influence of male defended oviposition sites on early embryo mortality in bullfrogs. Ecology 59(4):789-798.

KAM, Y.C., CHUANG, Z.S. \& YEN, C.F. 1996. Reproduction, oviposition site selection, and tadpole oophagy of an arboreal nester, Chirixalus eiffingeri (Rhacophoridae), from Taiwan. J. Herpetol. 30(1):52-59.
KIESECKER, J.M. \& SKELLY, D.K. 2000. Choice of oviposition site by gray treefrogs: the role of potential parasitic infection. Ecology 81(10):2939-2943.

LAURILA, A. \& AHO, T. 1997. Do female common frogs choose their breeding habitat to avoid predation on tadpoles? Oikos 78(3):585-591.

LAURILA, A. 1998. Breeding habitat selection and larval performance of two anurans in freshwater rock-pools. Ecography 21(5):484-494.

LIN, Y.S. \& KAM, Y.C. 2008. Nest choice and breeding phenology of an arboreal breeding frog, Kurixalus eiffingeri (Rhacophoridae), in a bamboo forest. Zool. stud. 47(2):129-137.

MARSH, D.M., RAND, A.S. \& RYAN, M.J. 2000. Effects of interpond distance on the breeding ecology of túngara frogs. Oecologia 122(4):505-513.

MARSH, D.M. \& BORRELL, B.J. 2001. Flexible oviposition strategies in túngara frogs and their implications for tadpole spatial distributions. Oikos 93(1):101-109.

MATSUSHIMA, N. \& KAWATA, M. 2005. The choice of oviposition site and the effects of density and oviposition timing on survivorship in Rana japonica. Ecol. Res. 20(1):81-86.

MITCHELL, N.J. 2002. Nest-site selection in a terrestrially breeding frog with protracted development. Aust. J. Zool. 50(3):225-235.

MORRIS, D.W. 2003. Toward an ecological synthesis: a case for habitat selection. Oecologia 136(1):1-13.

MOUSSEAU, T.A. \& FOX, C.W. 1998. The adaptive significance of maternal effects. Tree 13(10):403-407.

MURPHY, P.J. 2003a. Does reproductive site choice in a Neotropical frog mirror variable risks facing offspring? Ecol. Monogr. 73(1):45-67.

MURPHY, P.J. 2003b. Context-dependent reproductive site choice in a Neotropical frog. Behav. Ecol. 14(5):626-633.

PETRANKA, J.W., HOPEY, M.E., JENNINGS, B.T., BAIRD, S.D. \& BOONE, S.J. 1994. Breeding habitat segregation of wood frogs and American toads: the role of interspecific tadpole predation and adult choice. Copeia 1994(3):691-697.

RESETARITS Jr., W.J. \& WILBUR, H.M. 1989. Choice of oviposition site by Hyla chrysoscelis: role of predators and competitors. Ecology 70(1):220-228.

RESETARITS Jr., W.J. 1996. Oviposition site choice and life history evolution. Amer. Zool. 36(2):205-215.

RESETARITS Jr., W.J. 2005. Habitat selection behavior links local and regional scales in aquatic systems. Ecol. Lett. 8(5):480-486.

RIEGER, J.F., BINCKLEY, C.A. \& RESETARITS Jr., W.J. 2004. Larval performance and oviposition site preference along a predation gradient. Ecology 85(8):2094-2099.

RUDOLF, V.H.W. \& RÖDEL, M.O. 2005. Oviposition site selection in a complex and variable environment: the role of habitat quality and conspecific cues. Oecologia 142(2):316-325.

SEALE, D.B. 1982. Physical factors influencing oviposition by the woodfrog, Rana sylvatica, in Pennsylvania. Copeia 1982(3):627-635.

SKELLY, D.K. 2001. Distributions of pond-breeding anurans: an overview of mechanisms. Israel. J. Zool. 47:313-332.

SPIELER, M. \& LINSENMAIR, K.E. 1997. Choice of optimal oviposition sites by Hoplobatrachus occipitalis (Anura, Ranidae) in an unpredictable and patchy environment. Oecologia 109(2):184-199.

ZINA, J. 2006. Communal nests in Physalaemus pustulosus (Amphibia: Leptodactylidae): experimental evidence for female oviposition preferences and protection against desiccation. Amphibia-Reptilia 27(1):148-150. 